\title{
An Amperometric D-Amino Acid Biosensor Prepared with a Thermostable D-Proline Dehydrogenase and a Carbon Nanotube-Ionic Liquid Gel
}

\author{
Yuji Tani, ${ }^{* 1}$ Yukiko Itoyama, ${ }^{* 1}$ Kenichi NishI, ${ }^{* 1}$ Chikahiro WAdA, ${ }^{* 1}$ Yoshio Shoda, ${ }^{* 1}$ \\ Takenori Satomura, ${ }^{* 2}$ Haruhiko SakURaba, ${ }^{* 3}$ Toshihisa OHShIma, ${ }^{* 4}$ Yukako HaYaShI, ${ }^{* 1}$ \\ Tomoki YABUTANI, ${ }^{* 1 \dagger}$ and Junko MotonaKA*1 \\ *1 Department of Chemical Science and Technology, Institute of Science and Tecnhology, \\ The University of Tokushima, 2-1 Minami-josanjima, Tokushima 770-8506, Japan \\ *2 Department of Materials Science, Yonago National College of Technology, \\ 4448 Hikona, Yonago, Tottori 683-8506, Japan \\ *3 Department of Applied Biological Science, Faculty of Agriculture \& Graduate School of Agriculture, \\ Kagawa University, 2393 Ikenobe, Miki, Kita, Kagawa 761-0795, Japan \\ *4 Microbial Genetics Division, Institute of Genetic Resources, Faculty of Agriculture, Kyushu University, \\ 6-10-1 Hakozaki, Higashi, Fukuoka 812-8581, Japan
}

\begin{abstract}
Carbon nanotube (CNT) gel, which is composed of a mixture of single-wall CNT, an ionic liquid, and a thermostable D-proline dehydrogenase (D-Pro DH) immobilized electrode was utilized for the determination of D-amino acids (DAAs) in food samples. When a critical comparison with CNT, Ketjen Black (KB), and carbon powder (CP) was also carried out, the CNT/D-Pro DH immobilized electrode showed the highest sensitivity and the lowest detection limit of D-proline. In addition, the CNT/D-Pro DH immobilized electrode was applied to detection of DAAs in rice wine and vinegar samples. The concentrations of DAAs in rice wine and vinegar samples were $0.0210 \pm 0.0001$ and $0.55 \pm 0.05 \mathrm{mmol} \mathrm{L}^{-1}$, respectively.
\end{abstract}

(Received March 18, 2009; Accepted May 21, 2009; Published July 10, 2009)

\section{Introduction}

Recently, progress in analytical methods has revealed the biological and physiological roles of D-amino acids (DAAs). In particular, D-serine distributes widely in the brain of schizophrenic and Alzheimer sufferer's, and is suspected to be involved in several neurological diseases. ${ }^{1,2}$ It is expected that DAAs enter the body through foods. ${ }^{3}$ Therefore, it is important to analyze DAAs in food for clarifying the intake process. However, data of DAAs concentration in food samples has rarely been accumulated. It is known that the D-proline concentration increases in vinegars during aging, and this phenomenon has potential as an indicator for examining the aging process. ${ }^{4,5}$ Furthermore, amino acids in drinks affect their taste and aroma. ${ }^{6}$ Erbe and Bruckner have determined the concentration of DAAs in beer to evaluate beer quality.? However, conventional analytical methods for DAAs involve the use of such techniques as high-performance liquid chromatography, gas chromatography and amino acids analyzers, which are generally time-consuming processes. ${ }^{8-10}$

The electrochemical methods can be operated with small equipments, and running costs are generally low. We have already developed an amperometric DAAs sensor in which a thermostable D-proline dehydrogenase (D-Pro DH) immobilized the electrode surface within an agar gel membrane. ${ }^{11}$ The D-Pro

† To whom correspondence should be addressed.

E-mail: yabutani@chem.tokushima-u.ac.jp
DH/agar immobilized sensor could be simply prepared and detect D-proline up to $0.5 \mathrm{mmol} \mathrm{L}^{-1} .^{11}$ However, the D-proline concentrations in food samples, such as vinegar $\left(0.1 \mathrm{mmol} \mathrm{L}^{-1}\right)^{12}$ and alcohol drinks $\left(0.005 \mathrm{mmol} \mathrm{L}^{-1}\right),{ }^{7}$ are generally low. Thus, it is necessary to improve the sensitivity for the analysis of these samples. In order to improve the sensitivity of a D-Pro DH/agar immobilized electrode, we selected carbon materials, such as carbon nanotube (CNT), Ketjen Black (KB) and carbon powder (CP). Since carbon materials have a superior electric conductivity and a large surface area, they have been widely used in electrochemical biosensors. ${ }^{13-15}$ For example, KB and CP were utilized as electrode materials for a biofuel cell ${ }^{14}$ and a $\mathrm{pH}$ sensor. ${ }^{15}$ Furthermore, there have been a large number of applications of CNT for biosensors. For example, a CNT/glucose oxidase (GOx) doped polypyrrole immobilized electrode and a screen-printed electrode immobilized with CNT/horseradish peroxidase (HRP)/polysulfone were reported for glucose and $\mathrm{H}_{2} \mathrm{O}_{2}$ sensors, respectively. ${ }^{16,17}$ Moreover, Fukushima et al. recently developed a CNT gel, which was a mixture of a CNT and imidazolium ion based ionic liquids. ${ }^{18}$ The CNT gel has a large potential for electrode materials because of its large surface area and easy membrane-forming performance. The CNT and ionic liquid have been used for such purposes as the formation of nanoparticles, ${ }^{19}$ lubricant additive, ${ }^{20}$ actuator $^{21,22}$ and elastic conductor. ${ }^{23}$ To the best of our knowledge, the application of the CNT gel to a DAAs sensor has not been reported yet.

In the present work, we researched an effective carbon material for improving the sensitivity of the D-Pro DH/agar immobilized electrode. Carbon materials $(\mathrm{CNT}, \mathrm{KB}, \mathrm{CP})$ and ionic liquid 
modified electrodes were used for comparisons of the sensor performance. The electrochemical properties of carbon materials, such as the conductivity and the surface configuration, were characterized by electrochemical methods. Finally, we determined the DAAs concentration in rice wine and vinegar samples using a CNT/D-Pro DH immobilized electrode.

\section{Experimental}

\section{Reagents}

D-Pro DH was obtained using a method reported by Satomura et al. $^{24}$ Potassium chloride, 2,6-dichloroindophenol (DCIP), potassium dihydrogenphosphate $\left(\mathrm{KH}_{2} \mathrm{PO}_{4}\right)$, disodium hydrogenphosphate 12-water, agar, and 4-methyl-2-pentanone were of analytical grade and purchased from Kanto Chemical Co. (Tokyo, Japan). DCIP was used as an electron mediator. D-Proline was of analytical grade, and purchased from Wako Pure Chemical Industries (Osaka, Japan). Poly-vinylidene fluoride-cohexafluoropropylene (PVdF) was purchased from Aldrich (Milwakee, USA). An ionic liquid of 1-butyl-3methylimidazolium tetrafluoroborate $\left(\mathrm{BMIBF}_{4}\right)$ was purchased from Merck (Darmstadt, Germany). A single-wall carbon nanotube (SWCNT: average particle diameter of $1-2 \mathrm{~nm}$ ) was purchased from Science Laboratories (Tokyo, Japan). KB (average particle diameter of $39.5 \mathrm{~nm}$ ) was purchased from Ketjen Black International Co. (Tokyo, Japan). CP (average particle diameter of $45 \mu \mathrm{m}$ ) was purchased from Strem Chemicals (Newburyport, USA).

\section{Electrochemical measurement}

All electroanalytical experiments were performed by using a potentiostat BAS-100B/W (BAS Inc. USA) equipped with a three-electrode system. A carbon-based gel/D-Pro DH/agar immobilized glassy carbon (GC) electrode was used as a working electrode. A platinum wire and $\mathrm{a} \mathrm{Ag} / \mathrm{AgCl}$ electrode (saturated $\mathrm{KCl}$ ) were used as counter and reference electrodes, respectively. Cyclic voltammograms were normally recorded at a scan rate of $1 \mathrm{mV} \mathrm{s}^{-1}$. A timebase technique in which the applied potential was held constant at $200 \mathrm{mV}$ vs. $\mathrm{Ag} / \mathrm{AgCl}$ was used for the determination of D-proline at $10 \mathrm{~min}$ after the addition of D-proline. Chronocoulometry (CC) was used for calculating the electrode surface area. The electrode surface area was calculated from the electrical charge $(Q)$ in $1.0 \mathrm{mmol} \mathrm{L}^{-1}$ potassium ferrocyanide in a $\mathrm{CC}$ measurement (step range, from 0 to $500 \mathrm{mV}$; pulse time, $250 \mathrm{~ms}$ ) based on a Cottrell plot,

$$
Q=2 n F A c(D t / \pi)^{1 / 2} \quad(\text { Cottrell plot })
$$

$(Q$, charge amount; $n$, number of electron transfers; $F$, Faraday constant $\left(\mathrm{C} \mathrm{mol}^{-1}\right) ; A$, electrode surface area $\left(\mathrm{cm}^{2}\right) ; c$, concentration of potassium ferrocyanide $\left(\mathrm{mol} \mathrm{cm}^{-3}\right) ; D$, diffusion constant $\left(\mathrm{cm}^{2} \mathrm{~s}^{-1}\right) ; t$, time (s)).

According to the integrated Cottrell equation, the surface area in the oxidation can be estimated from the slope of $Q v s$. the square root of time $\left(t^{1 / 2}\right)$. DCIP was dissolved in a $0.067 \mathrm{~mol} \mathrm{~L}^{-1}$ phosphate buffer solution (PBS) at $\mathrm{pH}$ 8.0. Before an electrochemical measurement, nitrogen gas was passed into the PBS. The measurement temperature was usually set at $50^{\circ} \mathrm{C}$ using a temperature-controlled bath (Type TR-2A, As One Co., Osaka, Japan). The temperature of the analytical solution was measured with a thermosensor in a temperature-controlled bath.

\section{Preparation of the CNT gel and D-Pro DH immobilized electrode}

First, a GC electrode (electrode diameter, $3 \mathrm{~mm}$ ) was sequentially polished with 6 and $1 \mu \mathrm{m}$ of diamond paste and $0.05 \mu \mathrm{m}$ alumina powder. The electrode was then rinsed with pure water. SWCNT $(5 \mathrm{mg})$ and $\mathrm{BMIBF}_{4}(21 \mu \mathrm{L})$ were mixed with a mortar and $6.3 \mathrm{mg}$ of $\mathrm{PVdF}$ was added to the mixture. Then, $250 \mu \mathrm{L}$ of 4-methyl-2-pentanone was added to a mixture of the SWCNT/BMIBF 4 and PVdF. This mixture was then heated at $80^{\circ} \mathrm{C}$ in order to obtain the CNT gel. The prepared CNT gel was treated with an ultrasonic homogenizer prior to spin-coating on a GC electrode. The polished GC electrode was set on an electrode rotating machine for spin-coating with CNT gel and the agar gel with D-Pro DH. Twenty microliters of the CNT gel were dropped onto the surface of the electrode under rotation at $4000 \mathrm{rpm}$. Subsequently, $15 \mu \mathrm{L}$ of a solution containing $1.5 \%(\mathrm{w} / \mathrm{v})$ agar and 6.4 units of D-Pro DH heated at $80^{\circ} \mathrm{C}$ was dropped onto the surface of the CNT gel immobilized electrode under rotation at $4000 \mathrm{rpm}$. The electrode was cooled at room temperature for $15 \mathrm{~min}$ and used for an electroanalytical measurement after rinsing its surface with pure water. The KB and $\mathrm{CP}$ gel electrodes were prepared using a similar procedure and composition as in the preparation for the CNT gel.

The electric conductivity of the CNT gel, the KB gel and the $\mathrm{CP}$ gel was determined using a four-probe method (HewlettPackard, Type-34401A). ${ }^{25}$ A UV-visible spectrophotometer (Jasco Model V-570, Tokyo, Japan) was used to check the amount of D-Pro DH in agar gel immobilized on the surface of the electrodes. The amount of D-Pro DH was determined by previously described methods. ${ }^{11}$

We determined the DAAs concentration in rice wine (origin, Japan; alcohol component, 15.5\%; raw material, Japanese rice) and vinegar (origin, China; raw material, sweet rice) samples using the CNT/D-Pro DH immobilized electrode. Since vinegar samples generally include easily oxidized substances, such as ascorbic acid, they were treated by a pre-oxidation method in order to remove these substances in advance. The treatment, in which the sample volume was $1 \mathrm{~mL}$, consisted of electrolyzation with a Pt mesh electrode at $1300 \mathrm{mV} v s . \mathrm{Ag} / \mathrm{AgCl}$ for $1 \mathrm{~h} .{ }^{11}$

\section{Results and Discussion}

\section{Preparation of the CNT gel/D-Pro DH immobilized electrode}

The CNT/D-Pro DH immobilized electrode was characterized by cyclic voltammetry. The cyclic voltammograms were measured in a pH 8.0 PBS containing $0.5 \mathrm{mmol} \mathrm{L}^{-1}$ DCIP and $0.1 \mathrm{~mol} \mathrm{~L}^{-1} \mathrm{KCl}$. The voltammograms are shown in Fig. 1. The electrocatalytic oxidation current of DCIP was observed at about $-100 \mathrm{mV}$ vs. $\mathrm{Ag} / \mathrm{AgCl}$, and increased with the addition of $20 \mathrm{mmol} \mathrm{L}^{-1}$ D-proline. The modification of the CNT gel raised the electrocatalytic oxidation current by $3-5$ times in comparison with the conventional D-Pro DH/agar immobilized electrode. The electron-transferring process occurring on the surface of the electrode can be summarized as follows:

D-Pro DH-FAD + D-proline $\longrightarrow$ D-Pro DH-FADH $2+$ pyrroline-2-carboxylate,

D-Pro DH-FADH ${ }_{2}+$ DCIP $_{(\mathrm{Ox})} \longrightarrow$ D-Pro DH-FAD + DCIP (Red)

$\mathrm{DCIP}_{(\text {Red })} \longrightarrow \mathrm{DCIP}_{(\mathrm{Ox})}+2 \mathrm{H}^{+}+2 \mathrm{e}^{-}$. 


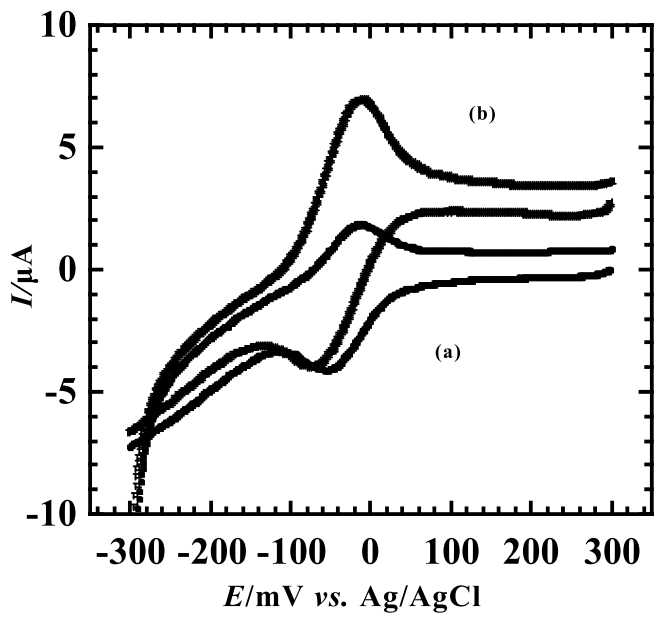

Fig. 1 Cyclic voltammograms of electrocatalytic oxidation of D-proline with a CNT/D-Pro DH immobilized electrode. The cyclic voltammograms were observed in a $\mathrm{pH} 8.0 \mathrm{PBS}$ containing $0.5 \mathrm{mmol}$ $\mathrm{L}^{-1}$ DCIP, $0.1 \mathrm{~mol} \mathrm{~L}^{-1} \mathrm{KCl}$, without (a) and with (b) $20 \mathrm{mmol} \mathrm{L}^{-1}$ D-proline at $50^{\circ} \mathrm{C}$. The scan rate was $1 \mathrm{mV} \mathrm{s}^{-1}$.

electrode was optimized. The CNT concentration strongly affects the current response and surface area of the electrode. First, we investigated the effect of the CNT concentration between 0.5 and $4.0 \%(\mathrm{w} / \mathrm{v})$ on the current response of D-proline. This result is shown in Fig. 2(a). The oxidation current upon the addition of $10 \mathrm{mmol} \mathrm{L}^{-1} \mathrm{D}$-proline was largest at $2.0 \%(\mathrm{w} / \mathrm{v})$, while it decreased above $2.0 \%(\mathrm{w} / \mathrm{v})$ of the CNT concentration. In a high CNT concentration range, a decrease in the current response might arise from inhibition of the diffusion of D-proline and DCIP because of the thick membrane of the CNT gel. A similar result was reported from a CNT/Nafion immobilized electrode for the determination of $p$-aminophenol. ${ }^{26}$ When the CNT/Nafion film was too thick, the current response of $p$-aminophenol decreased, because of a reduced mass transport. Consequently, the optimum CNT concentration was chosen as $2.0 \%(\mathrm{w} / \mathrm{v})$.

$\mathrm{PVdF}$ is a thermoplastic polymer, and is used as an immobilization matrix for the CNT gel on the surface of the electrode. ${ }^{23}$ We investigated the dependency of the PVdF concentration at between 1.5 and $3.5 \%(\mathrm{w} / \mathrm{v})$ on the current response of $10 \mathrm{mmol} \mathrm{L}^{-1} \mathrm{D}$-proline. This result is shown in Fig. 2(b). The maximum current response was observed at $2.5 \%$ $(\mathrm{w} / \mathrm{v})$, and its relative standard deviation (RSD) was less than $10 \%(n=3)$. For high PVdF concentrations above 3.0\% (w/v), the current response decreased and its RSD was over $20 \%$. The low current response and the smaller reliability of the high PVdF concentration were due to the formation of a thick, non-uniform membrane of CNT gel on the electrode, because of its high viscosity. Consequently, 2.5\% (w/v) PVdF was used as the optimum concentration in further measurements. The membrane thickness of the CNT gel on the electrode prepared in the optimal procedure was $1.1 \pm 0.4 \mu \mathrm{m}$.

Under the optimum conditions, the long-term stability of the CNT/D-Pro DH immobilized electrode was characterized by the current ratio. The current ratio was measured by the same CNT/D-Pro DH immobilized electrode. The initial current was defined as that measured on the first day of preparing the electrode. The CNT/D-Pro DH immobilized electrode was stored at room temperature under dry conditions. The long-term stability of the CNT/D-Pro DH immobilized electrode was tested by measuring every 7 days, and could keep $58 \pm 17 \%(n=3)$ of
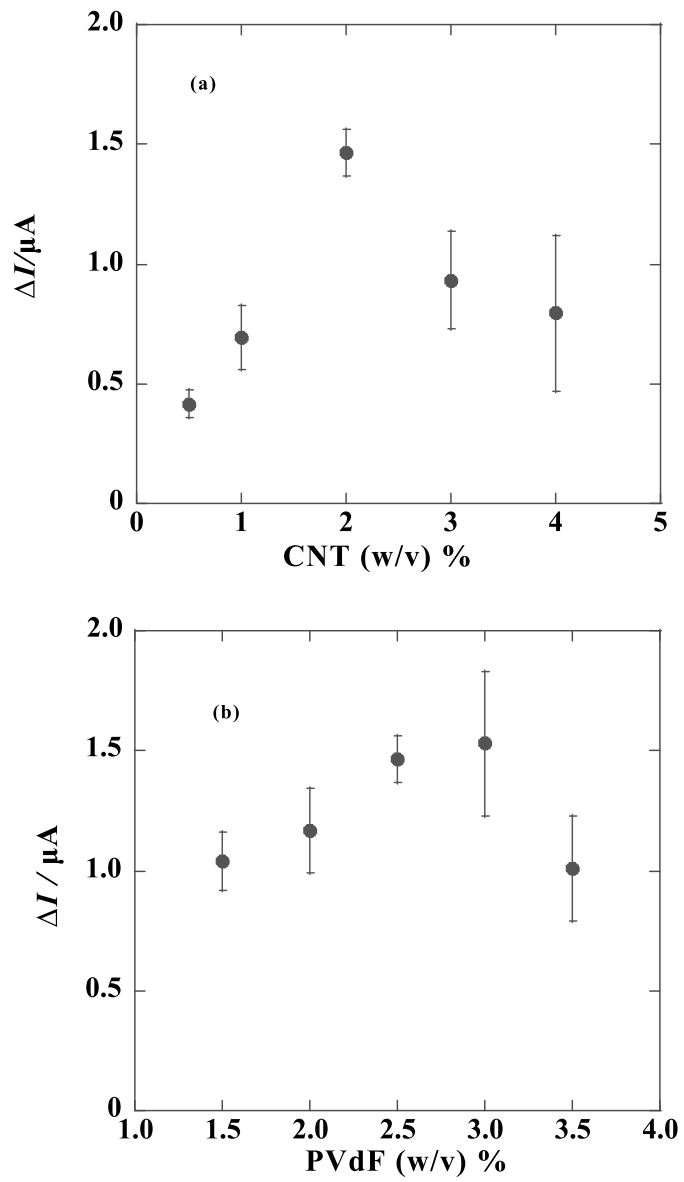

Fig. 2 Relationship between the CNT (a), PVdF concentration (b) during electrode preparation and the current response at the CNT/D-Pro DH immobilized electrode. The current response, $\Delta I$, was observed in $10 \mathrm{~mL}$ of pH 8.0 PBS containing $10 \mathrm{mmol} \mathrm{L}^{-1} \mathrm{D}$-proline, $0.5 \mathrm{mmol} \mathrm{L}^{-1}$ DCIP and $0.1 \mathrm{~mol} \mathrm{~L}^{-1} \mathrm{KCl}$ at $50^{\circ} \mathrm{C}$.

the initial current for 28 days. The long-term stability of the CNT/D-Pro DH immobilized electrode was lower than that of the D-Pro DH/agar immobilized electrode. ${ }^{11}$ This decrease in the long-term stability might arise from desorption of the immobilized D-Pro DH and the CNT gel layer because of the thick membrane on the surface of the electrode.

Comparison of the current response of D-proline with different carbon materials/D-Pro DH immobilized electrodes

We compared the current responses, in a $10 \mathrm{mmol} \mathrm{L}^{-1} \mathrm{D}$-proline solution, of each electrodes: CNT/D-Pro DH, KB/D-Pro DH, CP/D-Pro DH, and D-Pro DH-only immobilized electrode. The CNT/D-Pro DH immobilized electrode exhibited the maximum response $(1.9 \mu \mathrm{A})$, whereas, those of the KB/D-Pro $\mathrm{DH}$, the $\mathrm{CP}$ D-Pro DH and the D-Pro DH-only immobilized electrode were $1.5,0.75$ and $0.15 \mu \mathrm{A}$, respectively. The current responses with the carbon material/D-Pro DH immobilized electrodes were 7 - 20 times more than that of the D-Pro DH-only immobilized electrode. Calibration curbes of D-proline observed with the carbon material/D-Pro DH immobilized electrodes are depicted in Fig. 3. In addition, Table 1 summarizes the sensitivity and the detection limit of D-proline with these electrodes. The sensitivity was calculated as the slope of the calibration plot of D-proline at the electrode. The detection limits were estimated as triple the concentration corresponding to the background current observed at pH 8.0 PBS $(n=3)$. The CNT/D-Pro DH 


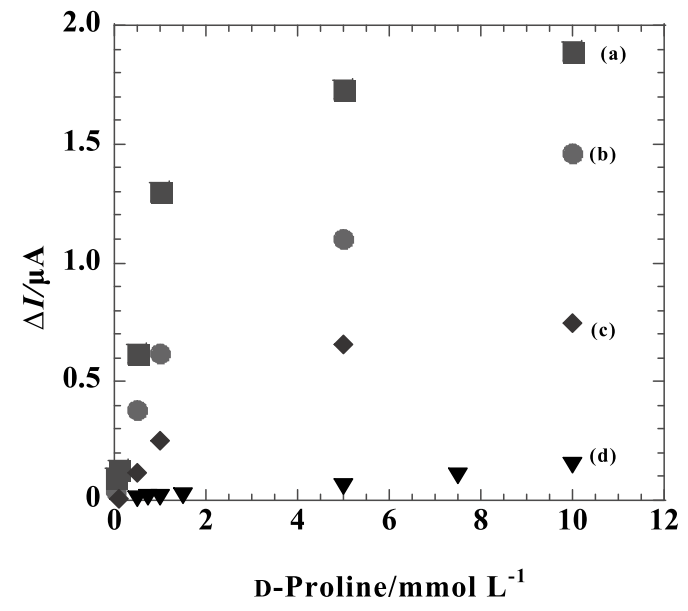

Fig. 3 Calibration plots for the oxidation of D-proline with CNT/D-Pro DH (a), KB/D-Pro DH (b), CP/D-Pro DH (c) and D-Pro DH-only immobilized electrode (d).

Table 1 Sensor performances with carbon material/D-Pro DH immobilized electrodes

\begin{tabular}{lllll}
\hline & $\mathrm{CNT}$ & $\mathrm{KB}$ & $\mathrm{CP}$ & \multicolumn{1}{c}{$\mathrm{GC}$} \\
\hline Detection limit/mmol L-1a & 0.002 & 0.01 & 0.05 & 0.5 \\
Sensitivity $/ \mu \mathrm{A} \mathrm{mmol} \mathrm{L}^{-1} \mathrm{~L}^{\mathrm{b}}$ & 1.3 & 0.59 & 0.12 & 0.014 \\
\hline
\end{tabular}

a. The detection limits were estimated based on triple the concentration corresponding to the background current observed in the $\mathrm{pH}$ 8.0 PBS $(n=3)$.

b. The sensitivity was defined as the slope of the calibration plot of D-proline at the electrode.

immobilized electrode exhibited the lowest detection limit and the highest sensitivity among these electrodes.

It was expected that the current response of the carbon-material immobilized electrodes would correlate with the surface area of the electrode, the electric conductivity of the carbon material and the amount of D-Pro DH on the surface of the electrode. These parameters were estimated, and the results are given in Table 2. The amounts of D-Pro DH onto the various carbon material immobilized electrodes showed little variation.

A modification of the carbon materials on the electrode increased the surface area of those electrodes, in comparison to the D-Pro DH-only immobilized electrode. Consequently, the surface area is deemed to be the major factor responsible for the increased current response. However, the surface area and the electric conductivity were also highest for the $\mathrm{KB}$ immobilized electrode. This result indicates that there were other factors that affected the current response. The CNT/D-Pro DH immobilized electrode shows the largest maximum current and sensitivity compared to other carbon material electrodes shown in Fig. 3. Thus, we also estimated the Michaelis-Menten constant $\left(K_{\mathrm{m}}\right)$ for the carbon material/D-Pro DH immobilized electrodes. The Michaelis-Menten constant $\left(K_{\mathrm{m}}\right)$ was calculated using a Lineweaver-Burk plot. The $K_{\mathrm{m}}$ of the CNT/D-Pro DH immobilized electrode was $0.83 \mathrm{mM}$, whereas those of KB/D-Pro DH, CP/D-Pro DH and D-Pro DH-only immobilized electrodes were $1.8,5.2$ and $7.9 \mathrm{mM}$, respectively. A large maximum current and low $K_{\mathrm{m}}$ generally indicate a high biological affinity between the enzyme and the substrate, and the CNT electrode shows a higher affinity to D-proline. In other words,
Table 2 Electrochemical properties and amount of D-Pro DH immobilized with carbon material/D-Pro DH immobilized electrodes

\begin{tabular}{|c|c|c|c|c|}
\hline & CNT & $\mathrm{KB}$ & $\mathrm{CP}$ & GC \\
\hline Surface area $/ \mathrm{cm}^{2}$ & 1.8 & 2.1 & 0.99 & 0.067 \\
\hline Conductivity $/ \mathrm{S} \mathrm{cm}^{-1}$ & 0.020 & 1.5 & 0.0030 & - \\
\hline Amount of D-Pro DH/mg ml- & 0.014 & 0.013 & 0.014 & 0.016 \\
\hline
\end{tabular}

the reactivity of the enzyme-substrate in the CNT/D-Pro DH immobilized electrode was higher than those of the other carbon material/D-Pro DH immobilized electrodes. The smaller $K_{\mathrm{m}}$ value for the CNT immobilized electrode is consistent with previous reports for CNT-modified enzyme sensors, such as CNT and lactate dehydrogenase (enzyme cofactor NAD) ${ }^{27}$ HRP (enzyme cofactor heme), ${ }^{17}$ and GOx (enzyme cofactor FAD) electrodes. ${ }^{28,29}$

Analytical application of CNT/D-Pro DH immobilized electrode In this study, the CNT/D-Pro DH immobilized electrode was applied to determine the concentration of DAAs in a rice wine and a vinegar samples. The measured value was defined as the total concentration of DAAs, since the D-Pro DH reacts with several DAAs, expect for D-proline. ${ }^{11}$ The concentration of DAAs in the rice wine and the vinegar samples were $0.0210 \pm$ 0.0001 and $0.55 \pm 0.05 \mathrm{mmol} \mathrm{L}^{-1}(n=3)$, respectively. These values were obtained using CNT gel modification. The concentration range of DAAs in vinegar reported in previous studies $\left(0.1-1.0 \mathrm{mmol} \mathrm{L}^{-1}\right)$ is approximately similar to our results. ${ }^{12,30,31}$ However, the concentration of DAAs in rice wine has been rarely reported, so we could not compare our data.

\section{Conclusions}

In this study, CNT gel was used as a modifier of D-Pro DH immobilized agar electrodes in order to improve their sensitivity to DAAs. The CNT gel membrane can be easily and uniformly formed on the electrode by spin-coating. The current response of D-proline was highest with the CNT/D-Pro DH immobilized electrode. The sensitivity of the CNT/D-Pro DH immobilized electrode was higher than that of $\mathrm{KB}, \mathrm{CP}$, and the conventional D-Pro DH-only immobilized electrodes. This result is due to an increase in the surface area of the electrode, and also by promoting the enzyme reaction, resulting from a modification of the CNT gel. The concentrations of DAAs in the rice wine and vinegar samples were obtained using a CNT/D-Pro DH immobilized electrode.

\section{Acknowledgements}

The present work was supported partly by Grant-in-Aid for Scientific Research (KAKENHI) (No. 19550091), from the Ministry of Education, Culture, Sports, Science and Technology of Japan.

\section{References}

1. T. Shinkai, V. De Luca, R. Hwang, D. J. Muller, M. Lanktree, G. Zai, S. Shaikh, G. Wong, T. Sicard, N. Potapova, J. Trakalo, N. King, C. Matsumoto, H. Hori, A. 
H. Wong, O. Ohmori, F. Macciardi, J. Nakamura, and J. L. Kennedy, Neuromol. Med., 2007, 9, 169.

2. S. Z. Wu, A. M. Bodles, M. M. Porter, W. S. Griffin, A. S Basile, and S. W. J. Barger, Neuroinflammation, 2004, 1, 2.

3. R. Patzold and H. Brückner, "D-Amino Acids: A New Frontier in Amino Acid and Protein Research-Practical Methods and Protocols", ed. R. Konno, H. Brückner, A. D'Aniello, G. Fisher, and N. Fujii, 2007, Nova Science Publishers Inc., New York, 337.

4. T. Erbe and H. Brückner, Z. Lebensm. Unters. Forsch. A, 1998, 207, 400.

5. T. Erbe and H. Brückner, Eur. Food Res. Technol., 2000, 211,6 .

6. H. Yu, Y. S. Ding, and S. F. Mou, Chromatographia, 2003, $57,721$.

7. T. Erbe and H. Bruckner, J. Chromatogr., A, 2000, 881 , 81.

8. M. Herrero, E. Ibanez, P. J. Martın-Alvarez, and A. Cifuentes, Anal. Chem., 2007, 79, 5071.

9. Y. Song, Y. Feng, M. H. LeBlanc, S. Zhao, and Y.-M. Liu, Anal. Chem., 2006, 78, 8121 .

10. M. G. Zampolli, G. Basaglia, F. Dondi, R. Sternberg, C. Szopa, and M. C. Pietrogrande, J. Chromatogr., A, 2007, 1150, 162.

11. Y. Tani, K. Tanaka, T. Yabutani, Y. Mishima, H. Sakuraba, T. Ohshima, and J. Motonaka, Anal. Chim. Acta, 2008, $619,215$.

12. J. Kohama, K. Saito, H. Sakamoto, Y. Iwasaki, R. Ito, M. Horie, and H. Nakazawa, Bunseki Kagaku, 2007, 56, 1019.

13. M. Zhang, K. Liu, L. Xiang, Y. Lin, L. Su, and L. Mao, Anal. Chem., 2007, 79, 6559.

14. Y. Kamitaka, S. Tsujimura, N. Setoyama, T. Kajino, and K. Kano, Phys. Chem. Chem. Phys., 2007, 9, 1793.

15. H. C. Leventis, I. Streeter, G. G. Wildgoose, N. S. Lawrence,
L. Jiang, T. G. J. Jones, and R. G. Compton, Talanta, 2004 63, 1039

16. J. Wang and M. Musameh, Anal. Chim. Acta, 2005, 539, 209.

17. S. Sanchez, M. Pumera, E. Cabruja, and E. Fabregas, Analyst, 2007, 132, 142.

18. T. Fukushima, A. Kosaka, Y. Ishimura, T. Yamamoto, T. Takigawa, N. Ishii, and T. Aida, Science, 2003, 300, 2072.

19. Y. S. Chun, J. Y. Shin, C. E. Song, and S.-G. Lee, Chem. Commun., 2008, 942.

20. B. Yu, Z. Liu, F. Zhou, W. Liu, and W. Liang, Mater. Lett., 2008, 62, 2967.

21. I. Takeuchi, K. Asaka, K. Kiyohara, T. Sugino, N. Terasawa, K. Mukai, T. Fukushima, and T. Aida, Electrochim. Acta, 2009, 54, 1762.

22. T. Sekitani, Y. Noguchi, K. Hata, T. Fukushima, T. Aida, and T. Someya, Science, 2008, 321, 1468.

23. T. Fukushima, K. Asaka, A. Kosaka, and T. Aida, Angew. Chem., Int. Ed., 2005, 44, 2410

24. T. Satomura, R. Kawakami, H. Sakuraba, and T. Ohshima, J. Biol. Chem., 2002, 277, 12861.

25. H. Tsuji, Y. Kawashima, H. Takikawa, and S. Tanaka, Polymer, 2007, 48, 4213.

26. Y. Cheng, Y. Liu, J. Huang, Y. Xian, W. Zhang, Z. Zhang, and L. Jin, Talanta, 2008, 75, 167.

27. Z. H. Gan, Q. Zhao, Z. N. Gu, and Q. K. Zhuang, Anal. Chim. Acta, 2004, 511, 239.

28. P. Du, B. Zhou, and C. Cai, J. Electroanal. Chem., 2008, 614,149

29. J. D. Qiu, W. M. Zhou, J. Guo, R. Wang, and R. P. Liang, Anal. Biochem., 2009, 305, 264

30. D. Carlavilla, M. V. M. Arribas, S. Fanali, and A. Cifuentes, Electrophoresis, 2006, 27, 2551.

31. S. Casal, M. B. Oliveira, and M. A. Ferreira, J. Chromatogr., A, 2000, 866, 221 